\title{
Trigeminocardiac reflex: beyond a skull base reflex
}

\author{
"The connection of the trigeminocardiac reflex and probably also of the \\ spinal cardiac reflex to oxygen-conserving reflexes opens the door to \\ new therapeutic options. One example is the important concept in \\ neurology of postconditioning.”
}

\section{Tumul Chowdhury', Nora Sandu ${ }^{2}$, Ricardo Gelpi ${ }^{3}$ \& Bernhard Schaller ${ }^{*, 2}$}

First draft submitted: 7 April 2017; Accepted for publication: 7 June 2017; Published online: 7 August 2017

The trigeminocardiac reflex (TCR) is a well-investigated brainstem reflex and was first described by the senior author in 1999 [1]. It has a wide array of manifestations including hemodynamic perturbations, arrhythmias, respiratory and gastric changes (see Figure 1) [2].

The TCR is mainly investigated as an autonomous reflex during skull base interventions [3-11] and its role in predicting the surgical outcomes is also explored [12-19]. The role of the TCR is not only limited to certain neurosurgical conditions and recently, it is also linked with various other surgical conditions, sleep disorders, sudden infant death syndrome and other interventions [20,21]. Similar to the TCR, recently Chowdhury and Schaller described the spinal cardiac reflex (SCR) [22] in spine surgeries that has also some similarities to the TCR; however, its mechanism is not yet fully elucidated.

This variance of its clinical occurrence, but also the fact that the TCR is not only an acute, but also chronic phenomena [23] is more than an intraoperative phenomenon. It seems that it is a (physiological) fundamental human behavior [24-26]. Therefore, this article provides an overview of this important reflex in clinical neurosciences and compiles all these consecutive concepts, for the first time, into a greater context.

Importance of the TCR in skull base \& spine surgery

The TCR has developed from a phenomenon during skull base surgery to a perioperative factor influencing the outcome after skull base surgeries/interventions [2,13,14,25,27-29]. This was already a milestone in neurosciences during the last 20 years. However, the exact mechanism of this is yet to be explored. Probable mechanisms of TCRinduced differential outcomes may include ischemia-induced, more manipulations of cranial nerve structures, more invasiveness of tumors, techniques, types of tumors or combinations of these factors [2,12]. In addition, many other factors including the effect of surgical positioning, type of anesthetics (intravenous vs volatiles), effect of blunt versus sharp dissection, and effect of other various surgical techniques were rarely studied

\section{KEYWORDS}

- bradycardia $\bullet$ oxygen conserving reflex $\bullet$ spinal cardiac reflex

- trigeminocardiac reflex 


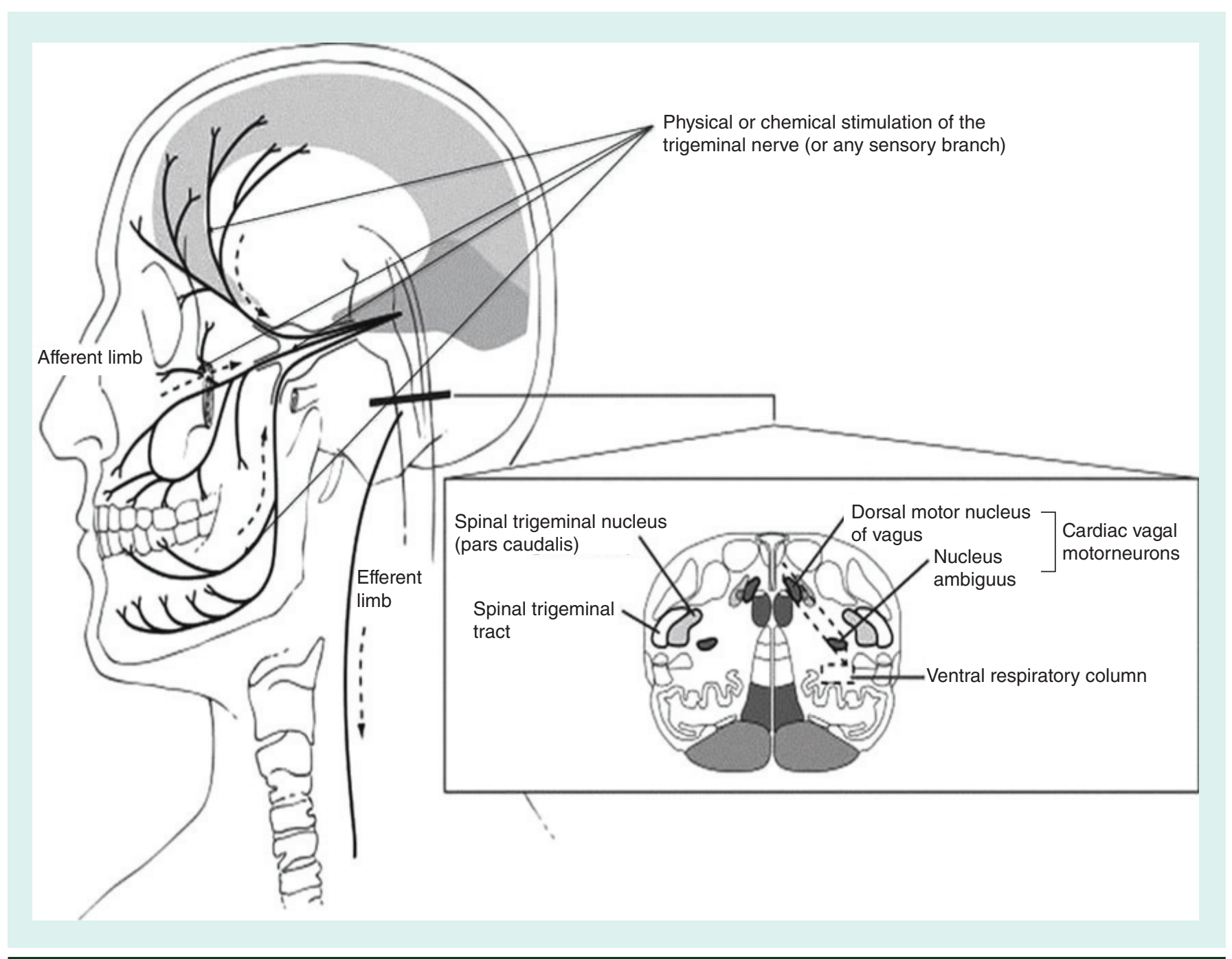

Figure 1. The trigeminocardiac reflex and its pathways. Figure taken with permission from [2].

in regards to the TCR phenomenon [15,17]. Future research should be directed toward adequately powered prospective randomized trials.

But now the story goes up a further step: A similar phenomenon may exist during spine surgeries as described by Chowdhury and Schaller [22]. It therefore seems that autonomous reflexes play a role in skull base and spine surgery. Whether or not, the similar connections at brainstem and efferent levels exist is a matter of further investigation. Why is that the case? We do not yet know the answer. But from examinations in sudden infant death [20], it seems that changes in the brainstem make the patients prone to the TCR and SCR.

\section{TCR: beyond neurosurgery}

There exists a complex interaction between the brain and heart. Various autonomic connections are part of this interaction. Similarly, the TCR, a well-studied reflex, is mainly investigated in acute surgical procedures and is considered as a (mild) exaggerated physiological response. Sometimes, it can be fatal. Our group demonstrated that the TCR phenomenon is not only limited to the clinical neurosurgical domain, it also has a possible influence on many conditions including bruxism, sudden death infant syndrome, obstructive sleep apnea, sudden death in adults, acute nonsurgical conditions (epistaxis) and forensic medicine [20,21]. Future investigations should reveal many more associations of TCR in various other neurological conditions. Most of these linkages postulate the acute nature of the TCR. However, our group recently explored the under-estimated chronic form of the TCR [11]. In this work, it seems plausible that chronic form of the TCR is mainly 
linked with the peripheral type. In addition, it is difficult to diagnose and presents initially with vague symptoms (nausea, dizziness). This information is very important for the general physicians, neurologists and other specialists to detect and diagnose this condition early. With all this new knowledge, the TCR has definitively shaped from a phenomenon that is mainly of interest for skull base surgeon and anesthetists to a wider audience.

There are some unanswered questions at present. Why is the peripheral subtype of the TCR commonly associated with the chronic form? What are the objective parameters to diagnose this condition? Lastly, what are the management options for the chronic TCR?

\section{New knowledge of TCR needs new definition}

We are now far away from the initial work of Schaller et al. in 1999 [1] that covered the TCR during surgery of vestibular schwannomas. There was a need to adapt and to improve the initial definition of the TCR, even so its genial structure that was the basis of this enormous development of this phenomenon should be maintained. Such work on the definition by Meuwly et al. [15] has also led to better and deeper understanding of the behavior of the TCR.

\section{Autonomous reflexes \& ischemic tolerance: the concept of oxygen- conserving reflex}

The concept of the oxygen-conserving reflex is investigated in context to the diving reflex and TCR [29]. The brain gets protected from the ischemia via two mechanisms. One is dependent on the rostroventrolateral (RVL) medullary connections and these are fast responders (within seconds) but are short lived. It has been seen (in animal experiments) that the trigeminal nerve stimulation (through the RVL medulla) produces intense cerebral vasodilation without increasing substantial oxygen consumption. In addition, the glucose uptake mechanism in the brain does not increase significantly [29]. On the other hand, the second mechanism (RVL medulla independent) is a slow responder but persists longer for few days to weeks. This mechanism can be beneficial in global ischemia as well [29].

In experimental studies (both human and animals) involving mandibular extension, it has been proven that the proprioceptive responses of the TCR modulate the cerebral pial vessel tone, diameter and also the redistribution of cerebral blood flow [28]. Therefore, it is plausible that this is a neuroprotective response; however, the clinical significance of this oxygen conserving mechanism is still unclear [29].

\section{Therapeutic aspects}

The connection of the TCR and probably also of the SCR to oxygen-conserving reflexes opens the door to new therapeutic options. One example is the important concept in neurology of postconditioning. Ischemic postconditioning is initially referred to when a stuttering reperfusion is performed immediately after reperfusion, for preventing ischemia/reperfusion injury in both myocardial and cerebral infarction. It has evolved into a concept that can be induced by a broad range of stimuli or triggers, and may even be performed as late as $6 \mathrm{~h}$ after focal ischemia and 2 days after transient global ischemia [30].

The peripheral TCR could also be such a clinically easy application for remote preconditioning. Whether or not some therapeutic advantages in stroke patients can be developed by using mandibular extension technique, cold stimulus of face or any other methods to stimulate the trigeminal sensory nerves is open for further research [30]. However, it would be an interesting domain to look into.

\section{Conclusion}

The TCR has evolved from a phenomenon to an important concept in clinical neuroscience. It seems that the SCR could also have this potential. This concept explains not only intraoperative or intrainterventional complications but also different neurological diseases. As part of this concept the oxygen-conserving reflex opens the window to different new therapeutic options.

\section{Financial \& competing interests disclosure}

The authors have no relevant affiliations or financial involvement with any organization or entity with a financial interest in or financial conflict with the subject matter or materials discussed in the manuscript. This includes employment, consultancies, honoraria, stock ownership or options, expert testimony, grants or patents received or pending, or royalties.

No writing assistance was utilized in the production of this manuscript. 


\section{References}

Papers of special note have been highlighted as:

- of interest; $\bullet \bullet$ of considerable interest

1 Schaller B, Probst R, Strebel S, Gratzl O. Trigeminocardiac reflex during surgery in the cerebellopontine angle. J. Neurosurg. 90 (2), 215-220 (1999).

-. Mentions the fundamental and classical description of the trigeminocardiac reflex (TCR). In its simplicity, this work is genial and has opened the window to this development of the TCR as it is now.

2 Schaller B, Cornelius JF, Prabhakar $\mathrm{H}$ et al. The trigemino-cardiac reflex: an update of the current knowledge. J. Neurosurg. Anesthesiol. 21(3), 187-195 (2009).

3 Schaller BJ. Trigeminocardiac reflex. J. Neurosurg. 107(1), 243 (2007).

4 Schaller BJ, Weigel D, Filis A, Buchfelder M Trigemino-cardiac reflex during transsphenoidal surgery for pituitary adenomas: methodological description of a prospective skull base study protocol. Brain Res. 1149, 69-75 (2007).

5 Schaller BJ, Rasper J, Filis A, Buchfelder M. Difference in functional outcome of ipsilateral tinnitus after intraoperative occurence of the trigemino-cardiac reflex in surgery for vestibular schwannomas. Acta Neurochir. (Wien). 150(2), 157-160 (2008).

6 Spiriev T, Kondoff S, Schaller B. Trigeminocardiac reflex during temporary clipping in aneurismal surgery: first description. J. Neurosurg. Anesthesiol. 23(3), 271-272 (2011).

- A relative simple, but groundbreaking work as it explains another important intraoperative sequela during skull base surgery.

7 Filis A, Schaller B, Buchfelder M. Trigeminocardiac reflex in pituitary surgery. A prospective pilot study. Nervenarzt. 79(6), 669-675 (2008).

8 Spiriev T, Sandu N, Kondoff S, Tzekov C, Schaller B. Tic and autonomic symptoms. J. Neurosurg. 116(6), 1397-1398 (2012).

9 Sandu N, Chowdhury T, Sadr-Eshkevari P et al. Trigeminocardiac reflex during cerebellopontine angle surgery: anatomical location as a new risk factor. Future Neurol. 10(1), 7-13 (2015).

10 Chowdhury T, Nöthen C, Filis A, Sandu N, Buchfelder M, Schaller B. Functional outcome changes in surgery for pituitary adenomas after intraoperative occurrence of the trigeminocardiac reflex: first description in a retrospective observational study. Medicine 94(37), e1463 (2015).

11 Chowdhury T, Schaller B. Chronic trigemino-cardiac reflex: an underestimated truth. Front. Neurol. 8, 22 (2017).

12 Chowdhury T, Sandu N, Meuwly C, Cappellani RB, Schaller B. Trigeminocardiac reflex: differential behavior and risk factors in the course of the trigeminal nerve. Future Neurol. 9(1), 41-47 (2014).

13 Sandu N, Sadr-Eshkevari P, Schaller BJ. Usefulness of case reports to improve medical knowledge regarding trigemino-cardiac reflex in skull base surgery. J. Med. Case Rep. 5(1), 1-3 (2011).

14 Chowdhury T, Mendelowitz D, Golanov E et al. Trigeminocardiac reflex: the current clinical and physiological knowledge. J. Neurosurg. Anesthesiol. 27(2), 136-147 (2015).

15 Meuwly C, Chowdhury T, Sandu N, Reck M, Erne P, Schaller B. Anesthetic influence on occurrence and treatment of the trigeminocardiac reflex: a systematic literature review. Medicine. 94(18), e807 (2015).

16 Sandu N, Chowdhury T, Schaller BJ. How to apply case reports in clinical practice using surrogate models via example of the trigeminocardiac reflex. J. Med. Case Rep. 10(1), 84 (2016).

- Introduces for the first time the model thinking into the TCR research.

17 Chowdhury T, Ahuja N, Schaller B. Severe bradycardia during neurosurgical procedure: depth of anesthesia matters and leads to a new surrogate model of the trigeminocardiac reflex: a case report. Medicine. 94(49), e2118 (2015).

18 Meuwly C, Chowdhury T, Sandu N, Schaller BJ. Meta-areas of the trigeminocardiac reflex within the skull base: a neuroanatomic 'thinking' model. J. Neurosurg. Anesthesiol. 28(4), 437-438 (2016).

19 Sandu N, Chowdhury T, Meuwly C, Schaller B. Trigeminocardiac reflex in cerebrovascular surgery: a review and an attempt of a predictive analysis. Expert Rev. Cardiovasc. Ther. 15(3), 203-209 (2017).

20 Singh GP, Chowdhury T, Bindu B, Schaller B. Sudden infant death syndrome - role of trigeminocardiac reflex: a review. Front.
Neurol. 7, 221 (2016).

21 Chowdhury T, Schaller B. The role of acute trigemino-cardiac reflex in unusual, non-surgical cases: a review. Front. Neurol. 7 , 186 (2016).

22 Chowdhury T, Schaller B. The negative chronotropic effect during lumbar spine surgery: a systemic review and aggregation of an emerging model of spinal cardiac reflex. Medicine. 96(1), e5436 (2017).

-• Groundbreaking work showing that a variant of the TCR also exists during spine surgery.

23 Bohluli B, Schaller B, Sadr-Eshkevari P, Momen-Heravi F. Trigeminal cardiac reflex: another all-or-none law? J. Oral Maxillofac. Surg. 68(11), 2922 (2010).

24 Nöthen C, Sandu N, Prabhakar H et al. Trigemino-cardiac reflex and antecedent transient ischemic attacks. Expert Rev. Cardiovasc. Ther. 8(4), 509-512 (2010).

25 Lemaitre F, Chowdhury T, Schaller B. The trigeminocardiac reflex - a comparison with the diving reflex in humans. Arch. Med. Sci. 11(2), 419-426 (2015).

26 Schaller B, Filis A, Sandu N, Rasper J, Noethen C, Buchfelder M. Trigemino-cardiac reflex may be refractory to conventional management in adults. Acta Neurochir. (Wien). 150 (9), 929-930 (2008).

27 Schaller B, Cornelius JF, Sandu N, Ottaviani G, Perez-Pinzon MA. Oxygen-conserving reflexes of the brain: the current molecular knowledge. J. Cell. Mol. Med. 13(4), 644-647 (2009).

28 Bohluli B, Schaller BJ, Khorshidi-Khiavi R, Dalband M, Sadr-Eshkevari P,Maurer P. , Trigeminocardiac reflex, bilateral sagittal split ramus osteotomy, gow-gates block: a randomized controlled clinical trial. J. Oral Maxillofac. Surg. 69(9), 2316-2320 (2011).

29 Schaller BJ, Sandu N, Cornelius JF, Filis A, Perez-Pinzon MA. Oxygen-conserving implications of the trigemino-cardiac reflex in the brain: the molecular basis of neuroprotection? Mol. Med. 15(5-6), 125-126 (2009).

30 Kerendi F, Kin H, Halkos ME et al. Remote postconditioning. Brief renal ischemia and reperfusion applied before coronary artery reperfusion reduces myocardial infarct size via endogenous activation of adenosine receptors. Basic Res. Cardiol. 100, 404-412 (2005). 